\title{
Efeito da geometria do bocal divergente sobre o empuxo de motor-foguete operando no vácuo
}

\author{
Diego Fernando Moro ${ }^{1}$ \\ Programa de Pós Graduação em Engenharia Mecânica (PG-Mec) \\ Universidade Federal do Paraná (UFPR), Curitiba, PR \\ Carlos Henrique Marchi ${ }^{2}$ \\ Departamento de Engenharia Mecânica \\ Universidade Federal do Paraná (UFPR), Curitiba, PR
}

\begin{abstract}
Resumo. Neste trabalho, simulações numéricas são usadas para uma melhor compreensão da parte divergente da tubeira de motores-foguete operando no vácuo. $\mathrm{O}$ código computacional utilizado nas simulações é chamado Mach2D 7.4, cujas características são: permite a resolução de escoamentos invíscido, laminar ou turbulento, em qualquer velocidade, emprega sistema de coordenadas não ortogonais, resolve as equações diferenciais utilizando o método dos volumes finitos. Entre os modelos citados, foi utilizado o escoamento invíscido para resolver o escoamento no interior da tubeira, usada em motores-foguete. O envelope da tubeira e as condições utilizadas neste trabalho são baseados em resultados experimentais de um artigo do AIAA Journal de um grupo de pesquisa do Jet Propulsion Laboratory (JPL) da NASA (National Aeronautics and Space Administration). Entre os 5 tipos de divergente testados, o divergente logarítmico foi o que resultou no maior coeficiente de empuxo. Para uma tubeira de comprimento constante, há um ângulo ótimo em relação a horizontal para a tubeira cônica e para a tubeira logarítmica que é de $20^{\circ}$. Foi encontrado também que os ângulos ótimos não se modificam com o comprimento da tubeira. Também foi possível obter um coeficiente de empuxo maior que o obtido pelo método de projeto de divergente supersônico de Anderson, para o mesmo comprimento de tubeira. Usando o ângulo ótimo foi possível obter um aumento de 1,78 a $2,91 \%$ no coeficiente de empuxo nos três comprimentos avaliados.
\end{abstract}

Palavras-chave. Tubeira, motor-foguete, efeito do divergente, escoamento bidimensional axissimétrico, CFD, propulsão de foguetes

\section{Introdução}

A tubeira de um motor-foguete é responsável por acelerar o escoamento dos gases e gerar desta forma o empuxo que impulsiona um foguete, isto é realizado em velocidades subsônicas restringindo a área da seção transversal ao escoamento, mas em velocidades

${ }^{1}$ difmoro@ufpr.br,difmoro@gmail.com

22marchi@ufpr.br,chmcfd@gmail.com 
supersônicas este efeito é revertido. O produto desta velocidade com o fluxo de massa são uma das duas parcelas da força de empuxo, como mostrado na Eq. 1:

$$
F=\underbrace{\dot{m} v}_{1}+\underbrace{\left(P_{s}-P_{e}\right) A_{s}}_{2}
$$

Sendo que, $\dot{m}$ é o fluxo de massa dos gases que saem do motor-foguete, $v$ é a velocidade de saída dos gases, $P_{s}$ é a pressão de saída dos gases, $P_{e}$ é a pressão externa e $A_{s}$ é a área de saída dos gases.

São mostradas 2 parcelas de força na Eq. 1: a primeira é a quantidade de movimento que os gases de saída exercem contra o motor-foguete e a segunda é a força a qual a pressão externa exerce contra ou a favor da área de saída dos gases. Em um ambiente com pressão nula (vácuo), o motor-foguete trabalha de forma mais eficiente.

Alguns autores propuseram formas de projetar tubeiras [1,7] que usam o método das características para resolver o escoamento bidimensional no interior do motor-foguete e projetar uma geometria ótima, chamada de geometria sino. Em relação a propriedades iniciais, a maioria dos autores admitem número de Mach unitário na garganta (como a tubeira de Anderson [1]) ou equações para encontrar a linha sônica e começando o método das caraterísticas a partir dali [7].

Neste trabalho o escoamento foi simulado com o programa chamado Mach2D 7.4, o escoamento modelado foi o invíscido (Equações de Euler).

Qual seria o efeito de diferentes geometrias (divergentes) na situação de vácuo na saída da tubeira? É possível obter uma maior força de empuxo com diferentes geometrias de tubeiras em comparação com a geometria sino ótima [1,7], ao impor algumas restrições? Este trabalho tem por objetivo principal responder a estas perguntas.

\section{Mach2D 7.4 e geometrias avaliadas}

A seguir são mostrados os modelos implementados e hipóteses do Mach2D 7.4.

\subsection{Modelos físico e numérico do Mach2D 7.4}

O programa Mach2D 7.4 considera um escoamento compressível, contínuo, não reativo, de entalpia e energia interna dependentes apenas da temperatura. A pressão, densidade e temperatura estão relacionados pela equação dos gases perfeitos. $\mathrm{O}$ escoamento é considerado axissimétrico, em regime permanente, sem radiação térmica e as paredes são adiabáticas.

As equações diferenciais que modelam o escoamento invíscido bidimensional são: a conservação da massa, conservação da quantidade de movimento linear (componentes z e r) e conservação da energia térmica. Estas equações são integradas em cada volume de controle e resolvidas usando o método dos volumes finitos [5]. Neste trabalho foram utilizadas as equações de Euler (escoamento invíscido), mas no programa é possível simular um escoamento laminar (Navier-Stokes) ou turbulento (modelo BaldwinLomax). O sistema iterativo foi encerrado quando o resíduo dos sistemas lineares 
chegou ao erro de máquina. O erro numérico também foi estimado e é mostrado juntamente com os resultados numéricos, estes resultados serão discutidos na seção 3 .

\subsection{Tubeira original da NASA}

As tubeiras utilizadas neste trabalho foram baseadas em uma tubeira experimental, mostrada em um artigo do AIAA Journal [2] de um grupo de pesquisa do Laboratório de Propulsão a Jato (Jet Propulsion Laboratory) da NASA (National Aeronautics and Space Administration). O esquema da tubeira original em forma axissimétrica está mostrado na Fig. 1, foram repartidas em cinco partes denotadas pelas cores.

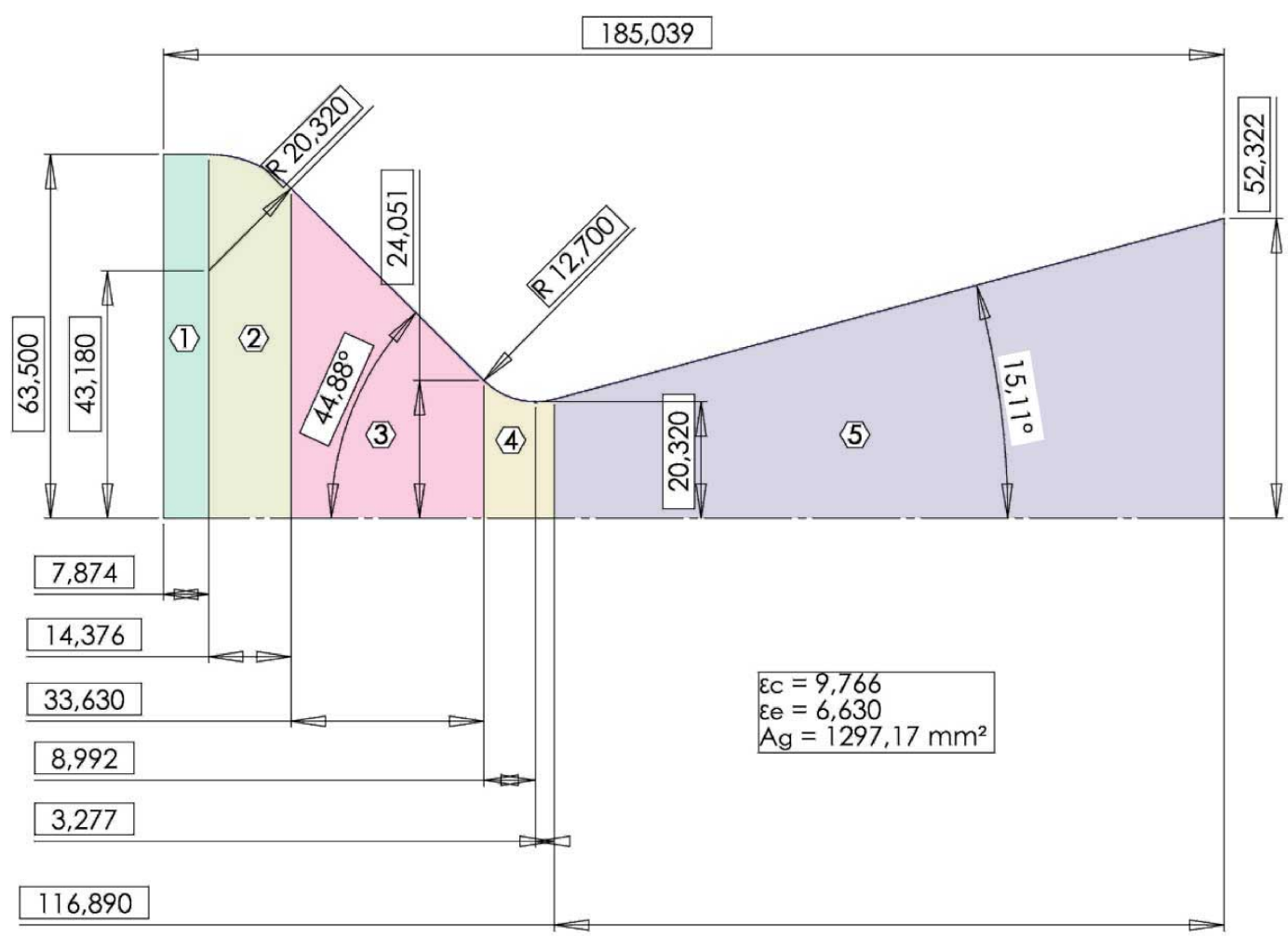

Figura 1 Esquema da tubeira original como mostrado no artigo [2].

Na Fig. 1: todas as medidas estão em mm, exceto as razões de expansão: $\varepsilon_{c}$ é a razão de compressão de áreas e $\varepsilon_{e}$ é a razão de expansão. Estes valores são razões entre as áreas de entrada e de saída pela área da garganta respectivamente, e a área da garganta é denotada por Ag, que é a área mínima.

Todos os resultados deste trabalho fixaram a geometria do convergente (partes 1 até a área mínima da parte 4) e os parâmetros físicos utilizados, parâmetros mostrados na Tab. 1. 
Tabela 1: Propriedades físicas do escoamento (fluido: ar atmosférico).

\begin{tabular}{|l|l|l|}
\hline Parâmetro & Valor & Unidades \\
\hline Constante de gás perfeito & 286,9 & $\mathrm{~J} /(\mathrm{kg} . \mathrm{K})$ \\
\hline Razão de calores específicos & 1,4 & adimensional \\
\hline Pressão de estagnação & 1725068,3 & $\mathrm{~Pa}$ \\
\hline Temperatura de estagnação & 833,33333 & $\mathrm{~K}$ \\
\hline
\end{tabular}

A geometria do divergente, a sua área de saída e seu comprimento foram variados para avaliar os seus efeitos na força de empuxo exercida pelo motor-foguete operando no vácuo.

\subsection{Tubeiras testadas}

Quatro tipos de geometria de divergente foram testadas: (1) logarítmico $\left(y=a * \log \left(b^{*} x\right)\right.$ ), (2) meio cosseno (apenas metade da função cosseno), (3) 1/4 seno (apenas um quarto da função seno) e (4) meia parabola ( $\left.y=a x^{2}+b x\right)$.

A tubeira sino (ótima) não pode ser simulada com a área de saída e o comprimento original da tubeira da NASA, porque estas são curvas 2D. Deve-se fixar ou o comprimento ou a razão de expansão.

\section{Resultados}

Neste trabalho o coeficiente de empuxo no vácuo é utilizado para definir o desempenho da tubeira, ele é definido por:

$$
C_{f v}=\frac{F_{v}}{P_{0} A_{g}}
$$

Sendo que $F v$ é a solução numérica da força de empuxo no vácuo, $P_{0}$ é a pressão de estagnação. Note que como a pressão de estagnação e a área da garganta são fixas, a comparação deste parâmetro de desempenho é semelhante à comparação da força de empuxo no vácuo.

\subsection{Efeito de diferentes geometrias no desempenho do motor, com comprimento e razão de expansão constantes}

A Tab. 2 resume os testes realizados e está apresentado o coeficiente de empuxo no vácuo (Cfv 2D) e a sua eficiência ( $\left.\mathrm{Cfv}^{*}\right)$. Note que a eficiência é definida como a razão entre a solução $2 \mathrm{D}$ e a solução do escoamento isentrópico $1 \mathrm{D}$. A razão de expansão foi mantida a mesma da tubeira original, como está mostrado na Fig. 1 que é de 6,63. O valor do coeficiente de empuxo para o escoamento isentrópico 1D é, neste caso, de 1,61355 . 
Tabela 2: Resumo dos testes realizados.

\begin{tabular}{|l|l|c|l|}
\hline \multicolumn{1}{|c|}{ Geometria } & \multicolumn{1}{c|}{ Cfv $^{*}$} & Cfv 2D & OBS \\
\hline Logaritmico & 0,97372 & $1,57115 \pm 0,00016$ & Utc \\
\hline $1 / 4$ seno & 0,97108 & $1,56688 \pm 0,00017$ & Utc \\
\hline Meia parabola & 0,970715 & $1,566296 \pm 0,000058$ & Utc \\
\hline Cônico (original) & 0,968427 & $1,562604 \pm 0,000080$ & Um \\
\hline Meio cosseno & 0,96203 & $1,55227 \pm 0,00018$ & Utc \\
\hline
\end{tabular}

Sendo que Cfv 2D é a solução numérica com o seu erro numérico estimado correspondente: Utc é um erro estimado bilateral e é baseado no estimador convergente [4] e Um é um erro estimado unilateral (leva em conta o sinal do erro estimado) e é baseado em Múltiplas Extrapolações de Richardson (MER) [6]. Estes resultados foram obtidos utilizando-se 7 malhas $(23 \times 10,46 \times 20$, até $1472 \times 640$ com refino constante de 2 em cada direção).

Como o erro estimado é pequeno em comparação com a solução, a próxima avaliação do efeito do divergente será realizada em uma única malha, 368x160 volumes de controle na direção axial e transversal respectivamente.

\subsection{Efeito de diferentes razões de expansão em tubeiras com comprimento constante}

A função que dá a forma ao divergente foi mantida, como o cônico, logarítmico ou meio cosseno, mas a razão de expansão foi variada de forma a gerar um ângulo com a horizontal de 10 a 30 graus com incrementos de 2 graus.

Os resultados destes testes são mostrados na Fig. 2 (esquerda). Observa-se que a geometria cônica possui um ângulo ótimo maior que 15 graus, o que a literatura considera o ótimo [3]. O ângulo ótimo encontrado para esta malha é de 20 graus, tanto para divergente cônico quanto para o divergente logarítmico.

\subsection{Efeito de diferentes razões de expansão ao variar o comprimento da tubeira}

Outro tema pesquisado foi se a tubeira sino ótima de Anderson, com a mesma razão de expansão que a avaliação anterior, teria mais ou o mesmo coeficiente de empuxo. Note que este tipo de geometria (sino de Anderson) é bidimensional, isto significa que se a razão de expansão for fixada, o comprimento está livre para modificar-se. Estes resultados (Fig. 2, direita) seguem a mesma tendência da curva de coeficiente de empuxo unidimensional, mas o comprimento das tubeiras se torna impraticável. O aumento foi de $185,039 \mathrm{~mm}$ (da tubeira original, onde o comprimento da parte divergente é de $120,167 \mathrm{~mm}$ ) para desde $196,072 \mathrm{~mm}$ até $419,562 \mathrm{~mm}$ na primeira e na última razão de expansão respectivamente. Se esta tubeira fosse utilizada numa missão 
especial, seria desperdiçada massa por ter uma maior área superficial e por consequência uma maior massa.
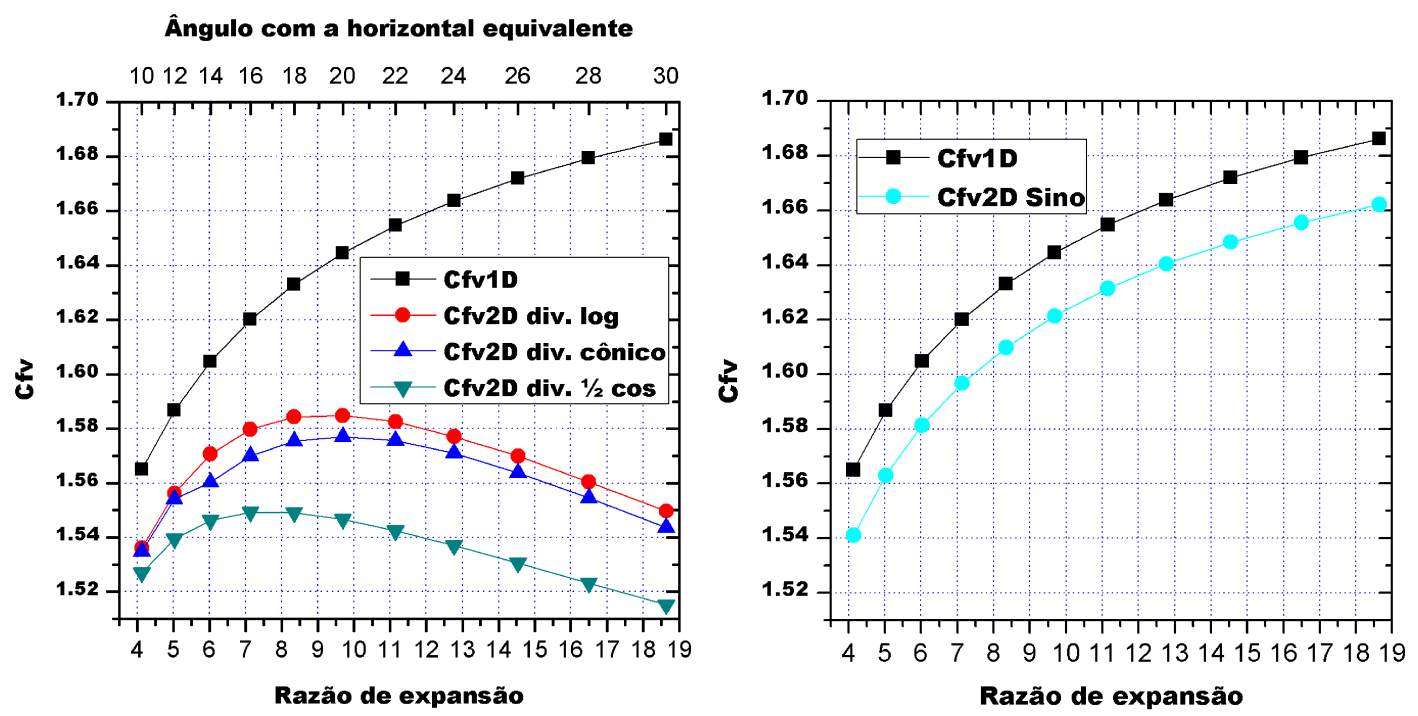

Figura 2: (Esquerda) Comparação do coeficiente de empuxo para as geometrias logarítmica, cônica e meio cosseno e (direita) resultados da tubeira sino de Anderson para as mesmas razões de expansão da Fig. 1 (mas com comprimentos distintos).

Com os três menores comprimentos de tubeira dados pelos testes com o divergente sino de Anderson, os três primeiros resultados da Fig. 2 direita, uma análise foi feita para avaliar o efeito do comprimento no coeficiente de empuxo. Por facilidade de simulação, apenas a geometria cônica foi utilizada.

O máximo do coeficiente de empuxo ocorre na razão de expansão correspondente ao ângulo cônico de $20^{\circ}$, o qual gerou um coeficiente de empuxo maior que o da tubeira sino de Anderson de mesmo comprimento. Este aumento no Cfv foi de 2,91; 2,29 e $1,78 \%$ para os 3 diferentes comprimentos utilizados: 196,08; 215,63 e 235,56 respectivamente. Temos portanto que o ângulo ótimo de 20 graus não se altera com o aumento no comprimento da tubeira.

\section{Discussão e conclusão}

Neste trabalho foi avaliado o efeito da parte divergente de tubeiras utilizadas em motores-foguete, isto foi realizado utilizando simulações numéricas invíscidas 2D. Foi avaliado por 3 diferentes formas: (1) fixando-se o comprimento e a razão de expansão e alterando apenas a geometria, (2) fixando-se o comprimento e alterando-se a razão de expansão, que depende do ângulo com a horizontal de 10 a 30 graus, e (3) usando 3 diferentes comprimentos e diferentes razões de expansão, este último tal como na segunda avaliação.

Baseado na avaliação (1), para as condições de operação (Tab. 1), a tubeira 
logarítmica é a geometria que gera o maior coeficiente de empuxo comparando com as outras geometrias testadas (Tab. 2).

Baseado na avaliação (2), o ângulo com a horizontal que gera o maior coeficiente de empuxo para as geometrias logarítmica e cônica é de 20 graus, e para a geometria meio cosseno é de 16 graus (Fig 2, esquerda) nas condições de operação desta tubeira.

Baseado na avaliação (3), o ângulo ótimo cônico não altera-se com o comprimento da tubeira.

\section{Agradecimentos}

Os autores agradecem o suporte financeiro do $\mathrm{CNPq}$ (Conselho Nacional de Desenvolvimento Científico e Tecnológico), AEB (Agência Espacial Brasileira) através do programa Uniespaço e a CAPES (Coordenação de Aperfeiçoamento de Pessoal de Nível Superior). O primeiro autor possui bolsa de doutorado da CAPES e o segundo é bolsista do CNPq.

\section{Referências}

[1] J. D. Anderson, Modern compressible flow: with historical perspective, 3 ed, pp. 397403, McGraw-Hill, (2003).

[2] L. H. Back, P. F. Massier and H. L. Gier, Comparison of measured and predicted flows through conical supersonic nozzles, with emphasis on the transonic region. AIAA Journal, vol. 3, n. 9, pp. 1606-1614, (1965).

[3] M. Farley and C. E. Campbell, NASA Technical Note D-293, National Aeronautics and Space Administration, Lewis Research Center, Ohio, (1960).

[4] C. H. Marchi, Verificação de soluções numéricas unidimensionais em dinâmica dos fluidos. Tese de Doutorado em Engenharia Mecânica. UFSC, (2001).

[5] C. H. Marchi e L. K. Araki, Relatório técnico do projeto CFD-10/UFPR: códigos Mach2D 6.1 e RHG2D 1.0, UFPR, Curitiba, (2009).

[6] M. A. Martins, Multiextrapolação de richardson com interpolação para reduzir e estimar o erro de discretização em CFD. Tese Doutorado em Engenharia Mecânica. UFPR, (2013).

[7] G. V. R. Rao, Exhaust Nozzle Contour for Optimum Thrust. Journal of Jet Propulsion, vol. 28, n.6, pp. 377-382, (1958). 